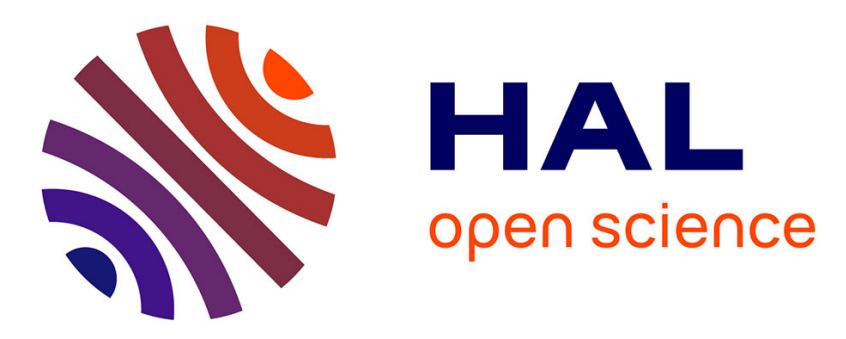

\title{
The V state of ethylene: valence bond theory takes up the challenge
}

Wei Wu, Huaiyu Zhang, Benoît Braïda, Sason Shaik, Philippe C. Hiberty

\section{To cite this version:}

Wei Wu, Huaiyu Zhang, Benoît Braïda, Sason Shaik, Philippe C. Hiberty. The V state of ethylene: valence bond theory takes up the challenge. Theoretical Chemistry Accounts: Theory, Computation, and Modeling, 2014, 133 (3), 10.1007/s00214-013-1441-x . hal-01627701

\section{HAL Id: hal-01627701 \\ https://hal.science/hal-01627701}

Submitted on 21 Nov 2017

HAL is a multi-disciplinary open access archive for the deposit and dissemination of scientific research documents, whether they are published or not. The documents may come from teaching and research institutions in France or abroad, or from public or private research centers.
L'archive ouverte pluridisciplinaire HAL, est destinée au dépôt et à la diffusion de documents scientifiques de niveau recherche, publiés ou non, émanant des établissements d'enseignement et de recherche français ou étrangers, des laboratoires publics ou privés. 


\title{
The $V$ state of ethylene: valence bond theory takes up the challenge
}

\author{
Wei Wu $\cdot$ Huaiyu Zhang $\cdot$ Benoît Braïda $\cdot$ \\ Sason Shaik · Philippe C. Hiberty
}

\begin{abstract}
The ground state and first singlet excited state of ethylene, so-called $N$ and $V$ states, respectively, are studied by means of modern valence bond methods. It is found that extremely compact wave functions, made of three VB structures for the $N$ state and four structures for the $V$ state, provide an $N \rightarrow V$ transition energy of $8.01 \mathrm{eV}$, in good agreement with experiment $(7.88 \mathrm{eV}$ for the $N \rightarrow V$ transition energy estimated from experiments). Further improvement to $7.96 / 7.93 \mathrm{eV}$ is achieved at the variational and diffusion Monte Carlo (MC) levels, respectively, VMC/DMC, using a Jastrow factor coupled
\end{abstract}

Dedicated to Professor Thom Dunning and published as a part of the special collection of articles celebrating his career upon his retirement.

\section{W. Wu $(\bowtie) \cdot H$. Zhang}

The State Key Laboratory of Physical Chemistry of Solid

Surfaces, Fujian Provincial Key Laboratory of Theoretical and Computational Chemistry, College of Chemistry and Chemical Engineering, Xiamen University, Xiamen 361005, Fujian, China e-mail: weiwu@xmu.edu.cn

B. Braïda ( $\square)$

Laboratoire de Chimie Théorique, CNRS, UMR 7616, UPMC Université Paris 06, C. 137, 4 Place Jussieu, 75252 Paris Cedex 05, France

e-mail: benoit.braida@upmc.fr

\section{S. Shaik $(\bowtie)$}

Institute of Chemistry and The Lise Meitner-Minerva Center for Computational Quantum Chemistry, Hebrew University

of Jerusalem, 91904 Jerusalem, Israel

e-mail: sason.shaik@gmail.com

P. C. Hiberty $(\square)$

Laboratoire de Chimie Physique, CNRS UMR8000, Bat. 349,

Université de Paris-Sud, 91405 Orsay Cédex, France

e-mail: philippe.hiberty@u-psud.fr with the same compact VB wave function. Furthermore, the measure of the spatial extension of the $V$ state wave function, $19.14 \mathrm{a}_{0}^{2}$, is in the range of accepted values obtained by large-scale state-of-the-art molecular orbitalbased methods. The $\sigma$ response to the fluctuations of the $\pi$ electrons in the $V$ state, known to be a crucial feature of the $V$ state, is taken into account using the breathing orbital valence bond method, which allows the VB structures to have different sets of orbitals. Further valence bond calculations in a larger space of configurations, involving explicit participation of the $\sigma$ response, with 9 VB structures for the $N$ state and 14 for the $V$ state, confirm the results of the minimal structure set, yielding an $N \rightarrow V$ transition energy of $7.97 \mathrm{eV}$ and a spatial extension of $19.16 \mathrm{a}_{0}^{2}$ for the $V$ state. Both types of valence bond calculations show that the $V$ state of ethylene is not fully ionic as usually assumed, but involving also a symmetryadapted combination of VB structures each with asymmetric covalent $\pi$ bonds. The latter VB structures have cumulated weights of 18-26\% and stabilize the $V$ state by about $0.9 \mathrm{eV}$. It is further shown that these latter VB structures, rather than the commonly considered zwitterionic ones, are the ones responsible for the spatial extension of the $V$ state, known to be ca. $50 \%$ larger than the $V$ state.

Keywords Valence bond Q Quantum Monte Carlo . $V$ state of ethylene - Breathing orbitals

\section{Introduction}

It often happens that valence bond (VB) theory provides compact and accurate descriptions of difficult test cases which, by contrast, necessitate long configuration 
expansions in the molecular orbital (MO) framework. As well-known examples, spin-coupled (SC) theory $[1,2]$ and generalized valence bond (GVB) theory $[3,4]$ are able to provide the most possible compact wave functions taking care of most of the static correlation energy in a molecule. Moreover, other VB-type methods include also dynamic correlation in a simple and lucid way [5-8]. Usually, dynamic correlation is retrieved in the MO framework by means of complete active space self-consistent-field approach (CASSCF) followed by a perturbative treatment (CASPT2), while VB methods may include dynamic correlation without increasing the number of VB structures, e.g., by using the breathing orbital effect [9-11].

The first singlet excited state of ethylene (so-called the $V$ state) is a notoriously difficult test case. Thus, even an extensive CASPT2 calculations, involving an all-valence $(12,12)$ active space, were found to be unsuccessful [12], and the correct description required more elaborate treatments. In the present paper, we pose the question whether VB theory can handle the challenge and still provide a compact but accurate description with a lucid account of electronic correlation? Taking up this challenge is our way of honoring Prof. Dunning who has significantly contributed to the revival of VB theory, among his other numerous achievements.

The $V$ state of ethylene, according to the Merer-Mulliken notation [13], is a singlet excited state of $B_{1 u}$ symmetry dominated by the $\pi_{\mathrm{u}} \rightarrow \pi_{\mathrm{g}} \mathrm{MO}$ configuration, which can be qualitatively represented in VB terms as a resonance between two zwitterionic structures, Scheme 1 . The $V$ state exhibits a broad UV absorption band with a maximum at $7.66 \mathrm{eV}$ [14]. For a long time now, the $V$ state poses an intriguing test case and exceptionally difficult to describe for theoreticians despite the small molecular size. Up to the late seventies, it was not even clear whether this state was of valence or Rydberg nature [15-19]. At the Hartree-Fock level, the $V$ state exhibited characteristics of a Rydberg state [15], owing to a very large spatial extent of the wave function as measured by an $\left\langle x^{2}\right\rangle$ expectation value of $42.1 \mathrm{a}_{0}^{2}$ ( $x$ being the axis orthogonal to the molecular plane), which is four times larger than the value of $11.9 \mathrm{a}_{0}^{2}$ computed for the ground state (the $N$ state) [20]. However, Bender et al. [20] and Buenker and Peyerimhoff [21] showed that wave functions computed with configuration interaction $(\mathrm{CI})$ are considerably more contracted than the

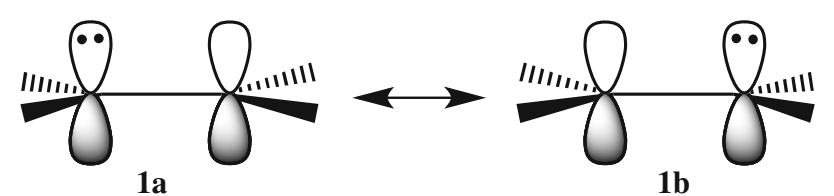

Scheme 1 Traditional representation of the $V$ ionic excited state of ethylene
Hartree-Fock one, so that the diffuse character of the state in the latter method is artificial. A few years later, McMurchie and Davidson [22] presented the first definitive theoretical evidence that the $V$ state is essentially a valence type. Thus, using a configuration selection scheme by classes of excitations in order to ensure a correct description of all correlation effects, involving the $\pi$ and $\pi^{*}$ electrons, these authors obtained the values $17.8 \mathrm{a}_{0}^{2}$ for $\left\langle x^{2}\right\rangle$ and $7.96 \mathrm{eV}$ for the $N \rightarrow V$ transition energy. Remarkably, McMurchie and Davidson found that including excitations of $\sigma \rightarrow \sigma^{*}$ type along with $\pi \rightarrow \pi^{*}$ ones are essential to give the $V$ state its correct spatial extension. This indicates that inclusion of dynamic electron correlation in the $\pi$ space must by accompanied by a concomitant reoptimization of the $\sigma$ orbitals [23, 24], a condition that is not met at the Hartree-Fock or CASSCF levels. It follows that even CASPT2 calculations, which are based on these latter levels, overestimate the energy of the $V$ state and place it close to the Rydberg $3 \mathrm{~d} \pi_{\mathrm{g}}$ configuration [25]. This energy proximity creates a spurious valence-Rydberg mixing, making the vertical $N \rightarrow V$ transition energy $\left(T_{\mathrm{e}}\right)$ artificially correct. As such, getting reasonable $T_{\mathrm{e}}$ values is not sufficient to validate a calculation, since improper orbital optimization can lead to spurious Rydberg character. A valid description of the $V$ state must display both an accurate $N \rightarrow V$ transition energy and an adequate value of spatial extent of the wave function, as measured by $\left\langle x^{2}\right\rangle$. Indeed, later computations by Buenker et al. [18, 19] obtained values of $19-20 \mathrm{a}_{0}^{2}$ for $\left\langle x^{2}\right\rangle$ and $8.05 \mathrm{eV}$ for $T_{\mathrm{e}}$, employing larger basis sets and MRSDCI configuration selection.

At this point, it is important to note that the calculated $T_{\mathrm{e}}$ value that best matches the experiment is not $7.66 \mathrm{eV}$, the maximum of the absorption band. The $T_{\mathrm{e}}$ value should be higher, if vibrational corrections were taken into account. On the basis of zero-point energy corrections, Davidson proposed that the best estimate for $T_{\mathrm{e}}$ should be $7.8 \mathrm{eV}$ [26]. More recently, Lasorne et al. [27] found a value of $7.92 \mathrm{eV}$ for $T_{\mathrm{e}}$ at a sophisticated MRCI level and then performed quantum dynamics simulation that reproduced the absorption spectrum with a maximum at $7.70 \mathrm{eV}$, only $0.04 \mathrm{eV}$ above the experimental value. As such, the theoretical $T_{\mathrm{e}}$ value that best matches experiment lies $0.22 \mathrm{eV}$ above the absorption maximum, giving rise to the value of $7.88 \mathrm{eV}$ as the best theoretical estimate.

Since the difficulty of calculating the $T_{\mathrm{e}}$ and $\left\langle x^{2}\right\rangle$ values arises from a lack of sufficient electron correlation at the orbital optimization step, various strategies have been devised to optimize the orbitals at a better level than Hartree-Fock or CASSCF. Roos et al. [28] used a multistate CASPT2 scheme, allowing the zero-order states to mix among themselves under the effect of dynamic correlation, and got a $T_{\mathrm{e}}$ value of $7.98 \mathrm{eV}$, with an $\left\langle x^{2}\right\rangle$ value 
in the range 18-20 $\mathrm{a}_{0}^{2}$. Krebs and Buenker performed an MRCI calculation in large basis set, including up to more than one million configurations state functions (CSFs), and estimated $T_{\mathrm{e}}$ in the range 7.90-7.95 eV [29]. An even more extensive MRCI calculation was performed by Müller et al. [30], including up to 80 million CSFs, yielding a $T_{\mathrm{e}}$ value of $7.7 \mathrm{eV}$. Both these MRCI calculations provide $\left\langle x^{2}\right\rangle$ values in the range $16.5-20 \mathrm{a}_{0}^{2}$. Still another strategy was used by Schautz and Filippi [31]. They performed diffusion Monte Carlo (DMC) calculations after optimization of the orbitals in the presence of the Jastrow factor at the VMC level. Their best $T_{\mathrm{e}}$ value was $7.92 \mathrm{eV}$, with $\left\langle x^{2}\right\rangle$ being $\sim 20 \mathrm{a}_{0}^{2}$. The quantum Monte Carlo (QMC) method was also used by Anderson and Goddard, in an endeavor to use a GVB determinantal part times a Jastrow factor as a DMC form of trial wave function. For the sake of cost-efficiency, the orbitals were taken from a previous bare GVB calculations and not reoptimized at the DMC level, as orbital reoptimization is a time-consuming part in QMC calculations. This strategy proved very successful for a number of excited states of ethylene and other molecules, but less so for the $V$ state of ethylene, leading to a $T_{\mathrm{e}}$ value of $8.27 \mathrm{eV}$ [32].

Recently, Angeli analyzed in detail the requirements for the correct description of the $V$ state of ethylene and more generally the ionic $\pi \rightarrow \pi^{*}$ excited states of polyenes, using qualitative VB reasonings [12]. The importance of the $\sigma-\pi$ mixing, also called $\sigma \pi$ correlation, was explained in terms of a dynamic response of the $\sigma$ framework to the field generated by the $\pi$ electrons in each ionic structure. In short, the $\sigma$ MOs follow the charge fluctuation in the $\pi$ system (represented as $\mathbf{1 a} \leftrightarrow \mathbf{1 b}$ in Scheme 1) by polarizing in a dynamic way so as to dampen the net charge on each carbon atom. Importantly, Angeli also showed that taking into account the latter effect induces a spatial contraction of the $\pi$ atomic orbitals (AOs), thus eliminating the spurious Rydberg character due to incomplete electron correlation. The strategy that was used to get an appropriate set of orbitals for CI was to optimize the orbitals in a self-consistent field manner using a restricted-activespace-SCF (RASSCF) calculation containing all the excitations describing the dynamic $\sigma$ polarization. Then, these orbitals were used in a CAS-CI $(2,2)$ or CAS$\mathrm{CI}(6,6)$, followed by a multistate perturbative treatment to second order. The procedure proved successful, with $\left\langle x^{2}\right\rangle$ values in the range $15.8-18.7 \mathrm{a}_{0}^{2}$ and $T_{\mathrm{e}}$ values of $7.65-7.80 \mathrm{eV}$.

It is clear from the above that the reasons for the early difficulties to describe the $V$ state are now well understood and that there exist well-defined strategies to obtain reasonable values for the diffuseness of this state as well as $N \rightarrow V$ transition energy in the MO-CI framework [12, 22,
33-35]. Still, the corresponding wave functions, which are constructed with large perturbative expansions, lack compactness and lucid interpretability, whereas the compact $\operatorname{CASSCF}(2,2)$ wave function is quite inaccurate. However, it has always been our experience that some ab initio VB methods, providing static and dynamic correlations [9-11, 36-38], are able to give quantitative results as well as physical insight by means of very compact wave functions, with a very small number of chemically lucid VB structures. Examples involve dissociation reactions of twoelectron bonds [9, 39] and three-electron bonds [40], reaction barriers [41, 42], transition energies [43], and so on [7]. Thus, in view of the difficulties encountered by many researchers and the elaborate strategies that had to be devised to describe the $V$ state of ethylene with MO-CI methods, we decided to address the problem by the use of $a b$ initio VB methods, with the aim of getting accurate values of $N \rightarrow V$ transition energies as well as diffuseness $\left\langle x^{2}\right\rangle$ values, from compact and insightful VB wave functions. Furthermore, we want the strategy for choosing the relevant VB structures to emerge from clear physical principles, without the need of preliminary large-scale calculations.

There are already some clues that this simple approach should be successful: (1) the effect of paramount importance to the accurate description of the $V$ state is the dynamic response of the $\sigma$ orbitals to the fluctuations of the $\pi$ electrons. This effect is very simply described by means of the breathing orbital valence bond (BOVB) method [911]. Furthermore, this effect can be easily turned off, allowing one to visualize and assess the effects of dynamic $\sigma \pi$ correlation on the size of orbitals and on the $N \rightarrow V$ transition energy. (2) BOVB, as well as quantum Monte Carlo valence bond methods (QMC-VB) [44-46], includes all the necessary dynamic correlation for both the $N$ and $V$ states, and (3) most of the MO-CI strategies that get an accurate description of the $V$ state are in fact based on qualitative VB reasonings.

Based on these considerations, modern VB methods can take up the challenge of reconciling compactness, accuracy, and physical insight in the calculation of the $V$ state of ethylene. The paper is organized as follows. First, the VB methods are briefly described in a theoretical section. Then, the VB structures are chosen, and finally, the results are presented and discussed.

\subsection{Theoretical methods}

\subsubsection{The VB wave function}

A many-electron system wave function, $\Psi$, is expressed in VB theory as a linear combination of Heitler-LondonSlater-Pauling (HLSP) functions, $\Phi_{K}$ in Eq. 1, 
$\Psi=\sum_{K} C_{K} \Phi_{K}$

where $\Phi_{K}$ corresponds to "classical" VB structures, and $C_{K}$ is the corresponding structural coefficients. An important feature of our VB calculations is that all the active orbitals, i.e., those that have varying occupancies in the VB structures, are strictly localized on a single atom, like in the classical VB method. This ensures a clear correspondence between the mathematical expressions of the VB structures and their physical meaning, ionic or covalent.

Several definitions exist for the weights of the VB structures [6]. The Coulson-Chirgwin formula [47], Eq. 2, is the equivalent of a Mulliken population analysis in VB theory:

$W_{K}=C_{K}^{2}+\sum_{L \neq K} C_{K} C_{L} S_{K L}$

Here, $S_{K L}$ is the overlap integral of two VB structures.

However, as this definition becomes meaningless when the overlaps are large, alternative definitions have also been proposed. Löwdin's scheme for symmetric orthogonalization [48] (Eq. 3)

$W_{K}^{L \ddot{w} w d i n}=\sum_{I J} S_{K I}^{1 / 2} C_{I} S_{K J}^{1 / 2} C_{J}$

is an attractive option, which leads to orthogonalized functions deviating as little as possible from the original set.

Another appropriate definition, which also remedies the overlap dependence problem, is the "inverse-overlap" Norbeck-Gallup formula [49] (Eq. 4), which is close to the normalized squares of coefficients:

$W_{K}^{I n v}=\frac{N C_{K}^{2}}{S_{K K}^{-1}}$

where $N$ is a normalization factor.

\subsubsection{The VB levels}

There are several computational approaches for VB theory at the ab initio level $[5,7]$. In the VB self-consistent field (VBSCF) procedure [50], both the VB orbitals and structural coefficients are optimized simultaneously to minimize the total energy. The breathing orbital valence bond method (BOVB) [9-11] improves the VBSCF accuracy without increasing the number of VB structures $\Phi_{K}$. This is achieved by allowing each VB structure to have its own specific set of orbitals during the optimization process, such that the orbitals can be different from one VB structure to the other. In this manner, the orbitals can fluctuate in size and shape so as to fit the instantaneous charges of the atoms on which these orbitals are located. As such, effectively, BOVB covers a much larger variational space than CASSCF/VBSCF, without losing the compactness of the wave function.

The orbitals in either VBSCF or BOVB are not restricted to be orthogonal, and thus, it is not guaranteed that orbitals that are localized on the same fragment will be independent of each other. However, since we always obtain a unique variationally optimized solution, wherein both orbitals and structural coefficients are fully optimized, the nonuniqueness of the orbitals, if happens, does not matter.

The BOVB method has several levels of sophistication [11]. Here, we chose the "SD-BOVB" level, whereby each doubly occupied active orbital is split into a pair of singly occupied orbitals accommodating a spin-pair, so as to account for the radial correlation of the electrons involved in the lone pair. Moreover, as indicated by the letter D (delocalized), the orbitals that do not belong to the active space are allowed to delocalize. The VBSCF and BOVB calculations have been performed using three different basis sets: (1) the standard 6-31G* basis set, (2) the correlation-consistent triple-zeta cc-pVTZ of Dunning et al. [51], and (3) a mixed basis set of triple-zeta + diffuse quality on the carbons, made of the aug-cc-pVTZ basis set [52] for carbons and of the cc-pVDZ basis set [51] for hydrogens. This latter basis set is referred to as aug-VTZ in this work.

\subsubsection{The VB-QMC levels}

Very recently, a mixed valence bond/quantum Monte Carlo (VB-QMC) method has been proposed which managed to provide high accuracy while keeping the full interpretative capabilities of classical valence bond methods [44-46]. The VB-QMC wave function we utilize here consists of a VBSCF determinant part multiplied by a Jastrow function that is included to account for electronic correlation. The Jastrow function is the same as in previous studies [44-46] and includes explicit two-body electron-electron, electronnucleus, and also three-body electron-electron-nucleus terms. In this study, the energies of the $N$ and $V$ states are computed by optimizing simultaneously Jastrow parameters, VB structural coefficients, and, in some specified cases, also orbitals. The variational Monte Carlo (VMC) optimization algorithm is used on the multi-structure wave function and separate atoms, respectively, by minimizing the total energy and a small percentage of variance $(1 \%)$ using the linear optimization method [46]. These VB-VMC wave functions are then projected onto the ground state using the diffusion Monte Carlo (DMC) algorithm under the fixed-node approximation, a procedure that recovers most of the remaining correlation effects that are missing at the VB-VMC level, while at the same time compensating 
Scheme 2 Set of VB structures for the $N$ and $V$ states in the $(2,2)$ space. The $\sigma-\mathrm{CC}$ bond is represented as a doubly occupied bonding $\mathrm{MO}$, which is polarized in opposite directions in $\mathbf{3 a}$ and $\mathbf{3 b}$ at the BOVB level

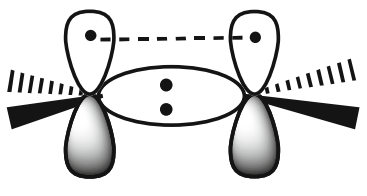

2

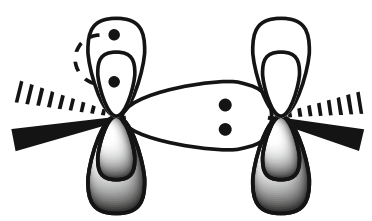

3a

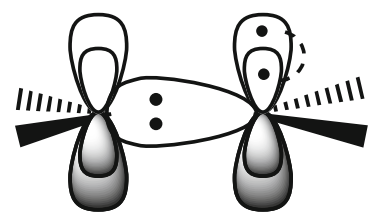

3b

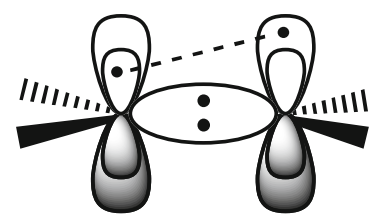

4a

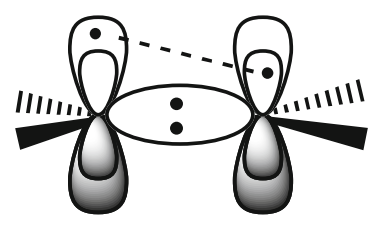

4b basis set deficiencies, and usually provides very accurate energy differences $[44,53]$. This latter method is referred to here as VB-DMC. For all VB-QMC calculations, a systematically convergent triple-zeta polarized basis set of Burkatzki et al. [54] for carbon, supplemented with the $s$ and $p$ diffuse functions taken from the standard aug-ccpVTZ basis set, and the double-zeta basis set of Burkatzki et al. for the hydrogen atom, was used together with their corresponding pseudo-potentials. The so-constructed basis set will be referred to here as ps-aug-VTZ.

All calculations were performed using the experimental equilibrium geometry of ethylene: $R_{\mathrm{CH}}=1.086 \AA$, $R_{\mathrm{CC}}=1.339 \AA, \angle \mathrm{HCH}=117.6^{\circ}$. The VBSCF and BOVB calculations were carried out with the Xiamen Valence Bond (XMVB) program [55-57]. All the QMC calculations were carried out using the CHAMP program [58].

\subsection{The VB structures set}

Restricting ourselves to an MO space of two orbitals and two electrons, so-called $(2,2)$ space, the description of the $N$ state of ethylene becomes straightforward: structure $\mathbf{2}$, in which the $\pi$ bond is fully covalent (Scheme 2), complemented with two ionic structures $\mathbf{3 a}$ and $\mathbf{3 b}$ that we define with split $\pi$ atomic orbitals (AOs) for full generality. For the $V$ state, the simplest way to get an elementary $\mathrm{VB}$ description is to expand the $\pi \pi^{*} \mathrm{MO}$ configuration into $\mathrm{VB}$ structures. Doing this in minimal basis set would yield the familiar picture $\mathbf{1 a} \leftrightarrow \mathbf{1 b}$ shown above in Scheme 1. However, the expansion in extended basis sets yields additional terms, because the $\pi$ and $\pi^{*}$ MOs are made from different AOs, $p_{\mathrm{a}}^{\prime}$ and $p_{\mathrm{b}}^{\prime}$ in $\pi^{*}$ being generally more diffuse than $p_{\mathrm{a}}$ and $p_{\mathrm{b}}$ in $\pi$ :

$\pi \propto\left(p_{\mathrm{a}}+p_{\mathrm{b}}\right)$

$\pi * \propto\left(p_{\mathrm{a}}^{\prime}-p_{\mathrm{b}}^{\prime}\right) ; p_{\mathrm{a}}^{\prime} \neq p_{\mathrm{a}}, p_{\mathrm{b}}^{\prime} \neq p_{\mathrm{b}}$

where the labels $a$ and $b$ refer to the left and right carbon atoms, respectively.
Using these orbital expressions and expanding $\pi \pi^{*}$ give rise to four VB structures instead of two, as shown in Eq. 6 below, dropping normalization constants:

$$
\begin{aligned}
\left|\pi \bar{\pi}^{*}-\bar{\pi} \pi^{*}\right| & =\left|\left(p_{\mathrm{a}}+p_{\mathrm{b}}\right)\left(\bar{p}_{\mathrm{a}}^{\prime}-\bar{p}_{\mathrm{b}}^{\prime}\right)-\left(\bar{p}_{\mathrm{a}}+\bar{p}_{\mathrm{b}}\right)\left(p_{\mathrm{a}}^{\prime}-p_{\mathrm{b}}^{\prime}\right)\right| \\
& =\left(\left|p_{\mathrm{a}} \bar{p}_{\mathrm{a}}^{\prime}\right|-\left|\bar{p}_{\mathrm{a}} p_{\mathrm{a}}^{\prime}\right|\right)-\left(\left|p_{\mathrm{b}} \bar{p}_{\mathrm{b}}^{\prime}\right|-\left|\bar{p}_{\mathrm{b}} p_{\mathrm{b}}^{\prime}\right|\right) \\
& -\left(\left|p_{\mathrm{a}} \bar{p}_{\mathrm{b}}^{\prime}\right|-\left|\bar{p}_{\mathrm{a}} p_{\mathrm{b}}^{\prime}\right|\right)+\left(\left|p_{\mathrm{b}} \bar{p}_{\mathrm{a}}^{\prime}\right|-\left|\bar{p}_{\mathrm{b}} p_{\mathrm{a}}^{\prime}\right|\right)
\end{aligned}
$$

where the terms in parentheses represent structures $\mathbf{3 a}, \mathbf{3 b}$, $\mathbf{4 a}$, and $\mathbf{4 b}$, respectively, in Scheme 2. In $\mathbf{3 a}$ and $\mathbf{3 b}$, one recognizes the zwitterionic structures analogous to $\mathbf{1 a}$ and $\mathbf{1 b}$, with the difference that the electrons pairs are split into different AOs, thus including some radial correlation. On the other hand, $\mathbf{4 a}$ and $\mathbf{4 b}$ are asymmetric covalent types, which have not been considered before. Their negative combination $(\mathbf{4} \mathbf{a}-\mathbf{4 b})$ has the right symmetry $\left(\mathrm{B}_{1 \mathrm{u}}\right)$ to contribute to the $V$ state and has the same constrained coefficient as $(\mathbf{3 a}-\mathbf{3 b})$ in $\pi \pi^{*}$ (Eq. 6). The importance or lack thereof of $4 \mathbf{a}$ and $\mathbf{4 b}$ as contributors to the $V$ state will be determined by the quantitative VB calculations below, in which the coefficients of all VB structures will be fully relaxed and optimized.

To calculate the $N \rightarrow V$ transition energy, it is necessary to calculate both the $N$ and $V$ states at a consistent level of accuracy. This will be done by describing the $N$ state with the three structures $\mathbf{2}, \mathbf{3 a}$, and $\mathbf{3 b}$ (these two latter ones will combine with a positive sign), while the $V$ state will involve the negative combinations $(\mathbf{3 a}-\mathbf{3 b})$ and $(\mathbf{4} \mathbf{a}-\mathbf{4} \mathbf{b})$. This set of five structures will be denoted as VBSCF-5 or BOVB-5, according to the level of VB theory. In all structures $\mathbf{2}-\mathbf{4}$ of the $(2,2)$ space, the $\sigma$ frame, made of the $\sigma-\mathrm{CC}$ and $\mathrm{CH}$ bonds, is described as a set of doubly occupied MOs. It should be noted that even this small $(2,2)$ space is sufficient to account for the $\sigma$ response to the fluctuation of $\pi$ electrons, provided the underlying $\sigma$ MOs are allowed to be different for different VB structures, as in the BOVB method. This effect is illustrated (in part) in Scheme 2 where the $\sigma-\mathrm{CC}$ bonding MOs are drawn as polarized in opposite directions in $\mathbf{3 a}$ and $\mathbf{3 b}$. 
Scheme 3 Set of VB structures for the $N$ and $V$ states in the $(4,4)$ space
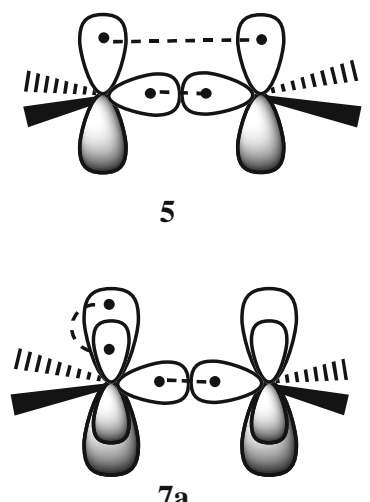

$7 \mathbf{a}$

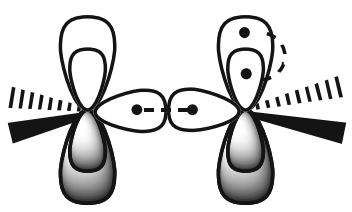

$7 b$

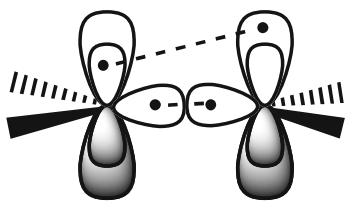

10a

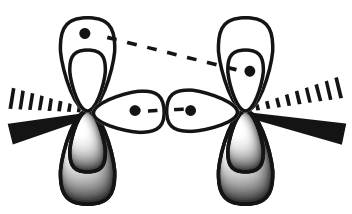

$10 \mathrm{~b}$

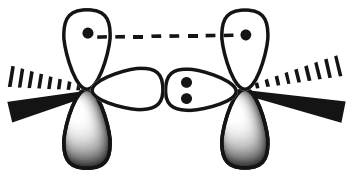

6a

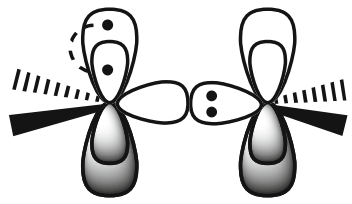

$8 \mathbf{a}$

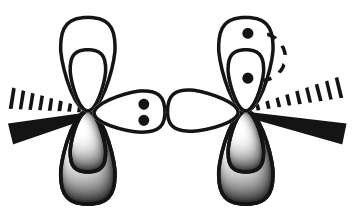

8b

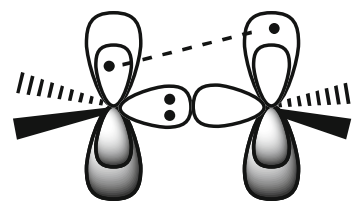

11a

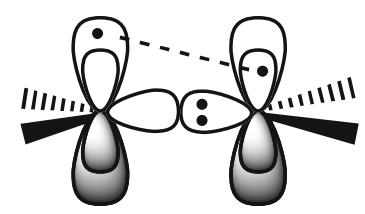

11b

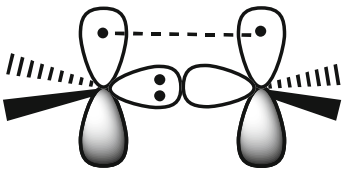

6b

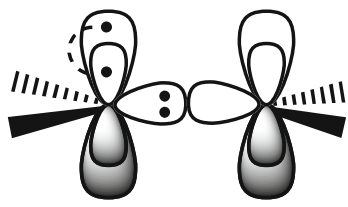

9a

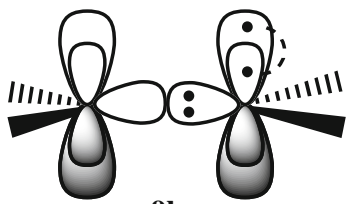

9b

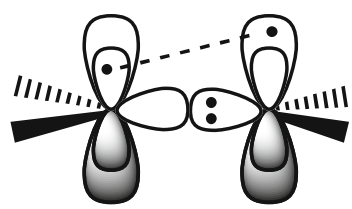

12a

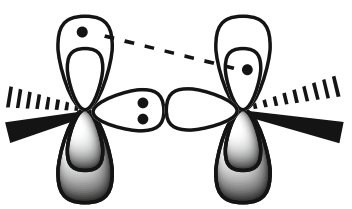

$12 b$
A more detailed way to allow for the $\sigma$ response to fluctuations of the $\pi$ electrons is to describe both the $\pi$ space and the $\sigma-\mathrm{CC}$ bond in a VB fashion. This can be achieved by extending the VB space for the $N$ and $V$ states to the 15 VB structures displayed in Scheme 3. Here, the $\sigma-\mathrm{CC}$ bond is not any more described as a doubly occupied MO, but as a combination of covalent and ionic structures. Hence, the $\sigma \pi$ correlation will be visualized as the difference in weights between structures $\mathbf{8 a}, \mathbf{8 b}$ versus $9 \mathbf{9 a}, \mathbf{9 b}$. The VB structure set in Scheme 3 will be denoted as VBSCF-15 or BOVB-15.

\section{Results}

\subsection{VB calculations in the $(2,2)$ space}

Although it is well known that basis sets involving diffuse basis functions are required for the description of states involving a dominant ionic component, it is interesting to start with smaller basis sets and then proceed to the largest.

Table 1 displays the results of calculations in the 6-31G* double-zeta basis set. For the $N$ state, all computational levels provide an $\left\langle x^{2}\right\rangle$ value in agreement with the accepted value, 11-12 $\mathrm{a}_{0}^{2}$ [20]. However, the calculated $\left\langle x^{2}\right\rangle$ values for the $V$ state appear to be much too small, around $12 \mathrm{a}_{0}^{2}$, showing that the $6-31 \mathrm{G}^{*}$ basis set is not appropriate to allow the $V$ state to be $50 \%$ more diffuse than the $N$ state, as it should. It is therefore not surprising that all $N \rightarrow V$ transition energies are largely overestimated in this basis set, even at the BOVB level (by $1.23 \mathrm{eV}$ in BOVB-5).

As can be seen in Table 2, the $\left\langle x^{2}\right\rangle$ values of the $V$ state are practically not improved in the larger cc-pVTZ basis set, which is of triple-zeta quality but still devoid of diffuse basis functions; however, the transition energies are improved and deserve some comments. The 
Table 1 The vertical excitation energy in $(2,2)$ active space, calculated by HF, CASSCF, and different VB methods with the 6-31G* basis set

\begin{tabular}{|c|c|c|c|c|c|}
\hline & \multicolumn{2}{|l|}{$N$ state } & \multicolumn{2}{|l|}{$V$ state } & \multirow{2}{*}{$\begin{array}{l}T_{\mathrm{e}} \\
(\mathrm{eV})\end{array}$} \\
\hline & E (a.u.) & $\begin{array}{l}\left\langle x^{2}\right\rangle \\
\left(a_{0}^{2}\right)\end{array}$ & E (a.u.) & $\begin{array}{l}\left\langle x^{2}\right\rangle \\
\left(a_{0}^{2}\right)\end{array}$ & \\
\hline $\begin{array}{l}\text { Hartree- } \\
\text { Fock }\end{array}$ & -78.030983 & 11.53 & & & \\
\hline \multicolumn{6}{|c|}{$(2,2)$ Active space } \\
\hline CASSCF & -78.059873 & 11.34 & -77.678207 & 12.08 & 10.39 \\
\hline VBSCF- $3^{\mathrm{a}}$ & -78.059878 & 11.34 & -77.657866 & 12.19 & 10.94 \\
\hline VBSCF- $5^{\mathrm{b}}$ & -78.059878 & 11.34 & -77.683298 & 12.08 & 10.25 \\
\hline $\mathrm{BOVB}-3^{\mathrm{a}}$ & -78.079418 & 11.29 & -77.718647 & 11.83 & 9.82 \\
\hline BOVB- $5^{\mathrm{b}}$ & -78.079418 & 11.29 & -77.744694 & 11.80 & 9.11 \\
\hline
\end{tabular}

${ }^{\mathrm{a}}$ Involves structures $\mathbf{2}, \mathbf{3 a}$ and $\mathbf{3 b}$ for the $N$ state, and $\mathbf{3 a}, \mathbf{3 b}$ for the $V$ state, as structure 2 cannot contribute to the $V$ state for symmetry reasons

${ }^{\mathrm{b}}$ Involves structures 2, 3a and $\mathbf{3 b}$ for the $N$ state, and 3a, 3b, 4a, 4b for the $V$ state. Structures $\mathbf{4 a}$ and $\mathbf{4 b}$ are not included in the $N$ state as their symmetric combination would be redundant with the structure 2

$N \rightarrow V$ transition energy is lower at the VBSCF-5 level than at the CASSCF one, although the latter configuration space contains all VB structures $\mathbf{2}-\mathbf{5}$ and more. This is because the coefficients of the VB structures in the CASSCF space are somewhat constrained, whereas they are fully optimized in VBSCF. Besides, the slight nonequivalence of CASSCF and VBSCF calculations even when both span the same variational space [6] has also been pointed out. ${ }^{1}$

The importance of structures $\mathbf{4 a}$ and $\mathbf{4 b}$ can be appreciated by performing a VB study in a smaller space, restricted to $\mathbf{2}, \mathbf{3 a}$, and $\mathbf{3 b}$. The results at the VBSCF and BOVB levels (referred to as VBSCF-3 and BOVB-3, respectively) show that removing $\mathbf{4 a}$ and $\mathbf{4 b}$ from the $\mathrm{VB}$ space causes a considerable increase in the transition energy relative to the reference VBSCF-5 and BOVB-5 levels, in both 6-31G* and cc-pVTZ basis sets, indicating that structures $\mathbf{4 a}$ and $\mathbf{4 b}$ stabilize the $V$ state by some 0.7 $0.9 \mathrm{eV}$. Lastly, the effect of dynamic correlation in the $\pi$ system on the transition energy, as measured by the BOVB-VBSCF difference, is seen to be quite large, ca. $0.9 \mathrm{eV}$ in cc-pVTZ basis set and $1.1 \mathrm{eV}$ in $6-31 \mathrm{G}^{*}$. These differences indicate that allowing the $\sigma$ response to fluctuations of the $\pi$ electrons is of importance, as will be analyzed in more details below. The best transition energy in these two basis sets amounts to $8.47 \mathrm{eV}$, at the BOVB-5/

\footnotetext{
${ }^{1}$ Slight differences between VBSCF and CASSCF calculations spanning the same variational space are due to the fact that the AOs that compose the MOs in CASSCF may be different in size and shape from one $\mathrm{MO}$ to the other, whereas the set of $\mathrm{AOs}$ is unique in VBSCF.
}

cc-pVTZ level. This last result, which is off by ca. $0.6 \mathrm{eV}$ relative to experiment, as well as the $\left\langle x^{2}\right\rangle$ diffuseness value which is found too small for the $V$ state, indicates that basis sets devoid of diffuse basis functions are inappropriate for the problem at hand, as already discovered long ago by Roos et al. [59, 60]. In accord, the rest of the study will be performed in a triple-zeta basis set augmented with diffuse basis functions on the carbon atoms, and a double-zeta basis set on the hydrogen atoms, so-called aug-VTZ (see Theoretical methods).

Table 3 displays results calculated in the aug-VTZ basis set, which in principle combines all the requirements in terms of flexibility and diffuseness to reliably describe an ionic state, which does not have a Rydberg character.

Inspection of Table 3 reveals that the $\left\langle x^{2}\right\rangle$ values of the ground state are practically unchanged relative to smaller basis sets and remain close to the generally accepted value of $12 \mathrm{a}_{0}^{2}$ at all levels. On the other hand, the $V$ state is affected by the augmented basis set. Thus, $V$ is found much too diffuse at the CASSCF level, as previously found by all researchers, with an $\left\langle x^{2}\right\rangle$ value of $25 \mathrm{a}_{0}^{2}$. Quite expectedly, about the same exaggerated spatial extension is found at the VBSCF-5 level, which lacks dynamic correlation like CASSCF, with the consequences that have been amply discussed in the past literature (vide supra). Thus, the fair agreement between experimental vertical energy and the $T_{\mathrm{e}}$ values that are found at these two levels, 8.25 and $8.26 \mathrm{eV}$, is artifactual and due to spurious mixing with a higher lying Rydberg configuration.

As shown in the Methods section, dynamic correlation is introduced by going from VBSCF to BOVB levels. As a first step, indicated as BOVB-5( $\pi$-only) level, the $\sigma$ orbitals remain common to all structures and only the $\pi$ orbitals are allowed to breathe for each of the structures $\mathbf{3 a}, \mathbf{3 b}, \mathbf{4 a}, \mathbf{4 b}$. It can be seen from Table 3 that there is practically no improvement at this level relative to VBSCF-5: The spatial extension diminishes only slightly, from 24.92 to $24.22 \mathrm{a}_{0}^{2}$, and the $N \rightarrow V$ transition energy is nearly unchanged. In a second step, we allow all orbitals to be different for different structures, at the BOVB-5 level. Here, the $\sigma$ response to the fluctuation of $\pi$ electrons in the $V$ state is turned on, and the change relative to CASSCF and VBSCF-5 is now clearly significant. The spatial extension of the wave function goes down to $19.14 \mathrm{a}_{0}^{2}$, quite in the range of accepted $\left\langle x^{2}\right\rangle$ values from high-level calculations. This result nicely illustrates the internal contraction of the wave function under the influence of dynamic correlation and also shows that essentially all the dynamic correlation that is attached to the description of the $\pi$ system in the $V$ state is made of the $\sigma$ response to the fluctuation of $\pi$ electrons. Another way of visualizing the contraction of the wave function is by comparing the different sizes of the $\pi$ AOs [12], calculated at the $\operatorname{CASSCF}(2,2)$ level (devoid of 
Table 2 Vertical excitation energies in $(2,2)$ active space, calculated by HF, CASSCF, and different VB methods with the cc-pVTZ basis set

\begin{tabular}{|c|c|c|c|c|c|}
\hline & \multicolumn{2}{|l|}{$N$ state } & \multicolumn{2}{|l|}{$V$ state } & \multirow[t]{2}{*}{$T_{\mathrm{e}}(\mathrm{eV})$} \\
\hline & E (a.u.) & $\left\langle x^{2}\right\rangle\left(\mathrm{a}_{0}^{2}\right)$ & E (a.u.) & $\left\langle x^{2}\right\rangle\left(\mathrm{a}_{0}^{2}\right)$ & \\
\hline $\begin{array}{l}\text { Hartree-Fock } \\
(2,2) \text { Active space }\end{array}$ & -78.063239 & 11.84 & & & \\
\hline CASSCF & -78.090945 & 11.58 & -77.741633 & 13.27 & 9.51 \\
\hline VBSCF- $3^{a}$ & -78.091235 & 11.59 & -77.713055 & 12.97 & 10.29 \\
\hline VBSCF- $5^{\mathrm{b}}$ & -78.091235 & 11.59 & -77.746673 & 13.32 & 9.38 \\
\hline BOVB- $3^{a}$ & -78.113076 & 11.71 & -77.766555 & 12.28 & 9.43 \\
\hline BOVB- $5^{b}$ & -78.113076 & 11.71 & -77.801926 & 12.72 & 8.47 \\
\hline
\end{tabular}

${ }^{a}$ Involves structures $\mathbf{2}, \mathbf{3 a}$, and $\mathbf{3 b}$ for the $N$ state, and $\mathbf{3 a}, \mathbf{3 b}$ for the $V$ state, as structure $\mathbf{2}$ cannot contribute to the $V$ state for symmetry reasons

${ }^{\mathrm{b}}$ Involves structures $\mathbf{2}, \mathbf{3 a}$, and $\mathbf{3 b}$ for the $\mathrm{N}$ state, and $\mathbf{3 a}, \mathbf{3 b}, \mathbf{4 a}, \mathbf{4 b}$ for the $V$ state. Structures $\mathbf{4 a}$ and $\mathbf{4 b}$ are not included in the $N$ state as their symmetric combination would be redundant with structure 2

dynamic correlation), shown in Fig. 1a, versus the dynamically correlated BOVB-5 level, Fig. 1b. First, it can be seen that the $p_{\mathrm{a}}$ and $p_{\mathrm{a}}^{\prime}$ AOs that are, respectively, part of the $\pi$ and $\pi^{*}$ MOs arising from the CASSCF calculations are very different in size, $p_{\mathrm{a}}^{\prime}$ being much more diffuse than $p_{\mathrm{a}}$ (Fig. 1a). Second, it clearly appears by comparing Fig. 1a, b that going from CASSCF to BOVB-5 induces a strong contraction of the $p_{\mathrm{a}}^{\prime}$ AOs. As an effect of $\sigma \pi$ dynamic correlation, the latter AOs have somewhat different shapes in $\mathbf{3 a}, \mathbf{3 b}$ versus $\mathbf{4 a}, \mathbf{4 b}$, but they have about the same spatial extension, quite smaller than at the CASSCF level.

Another nice feature of the BOVB-5 level is an excellent value of the $N \rightarrow V$ transition energy, $8.01 \mathrm{eV}$, only $0.13 \mathrm{eV}$ above the recommended value. This good $T_{\mathrm{e}}$ value together with a correct spatial extension of the wave

Table 3 The vertical excitation energy in $(2,2)$ active space, calculated by HF, CASSCF, and different VB methods with the augVTZ basis set

\begin{tabular}{|c|c|c|c|}
\hline$N$ state & & $V$ state & \\
\hline E (a.u.) & $\begin{array}{l}\left\langle x^{2}\right\rangle \\
\left(\mathrm{a}_{0}^{2}\right)\end{array}$ & E (a.u.) & $\begin{array}{l}\left\langle x^{2}\right\rangle \\
\left(\mathrm{a}_{0}^{2}\right)\end{array}$ \\
\hline
\end{tabular}

\begin{tabular}{llllll}
\hline $\begin{array}{l}\text { Hartree-Fock } \\
(2,2) \text { Active }\end{array}$ & -78.059607 & 12.01 & & & \\
space & & & & & \\
CASSCF & -78.087096 & 12.05 & -77.783590 & 25.08 & 8.26 \\
VBSCF-3 $^{\mathrm{a}}$ & -78.087129 & 11.68 & -77.733637 & 14.07 & 9.69 \\
VBSCF-5 $^{\mathrm{b}}$ & -78.087129 & 11.68 & -77.783951 & 24.92 & 8.25 \\
BOVB-5 $_{(\pi-\text { only })^{\mathrm{b}}}$ & -78.089552 & 11.73 & -77.786128 & 24.22 & 8.26 \\
BOVB-3 $^{\mathrm{a}}$ & -78.111200 & 11.64 & -77.782916 & 13.04 & 8.93 \\
BOVB-5 $^{\mathrm{b}}$ & -78.111200 & 11.64 & -77.816658 & 19.14 & 8.01 \\
\hline
\end{tabular}

${ }^{\text {a }}$ Involves structures $\mathbf{2}, \mathbf{3 a}$ and $\mathbf{3 b}$ for the $N$ state, and $\mathbf{3 a}, \mathbf{3 b}$ for the $V$ state, as structure $\mathbf{2}$ cannot contribute to the $V$ state for symmetry reasons

${ }^{\mathrm{b}}$ Involves structures 2, 3a and $\mathbf{3 b}$ for the $N$ state, and 3a, 3b, 4a, 4b for the $V$ state. Structures $\mathbf{4 a}$ and $\mathbf{4 b}$ are not included in the $N$ state as their symmetric combination would be redundant with the structure $\mathbf{2}$ function shows that compact VB functions with only three configurations for the $N$ state and four ones for the $V$ state are able to capture all the physics of the $N \rightarrow V$ excitation in ethylene, whereas many more configurations are required in the MO framework to reproduce the same effects.

It is interesting at this point to estimate the effect of $\sigma \pi$ dynamic correlation or polarization on the $T_{\mathrm{e}}$ value. One could be tempted to simply take it as the VBSCF- 5 versus BOVB-5 energy difference, i.e., $0.24 \mathrm{eV}$ (entries 4 vs. 7 in Table 3). However, one must recall that the relatively low $T_{\mathrm{e}}$ value at the VBSCF-5 (or CASSCF) level is artifactual and due to spurious valence-Rydberg mixing. It is therefore
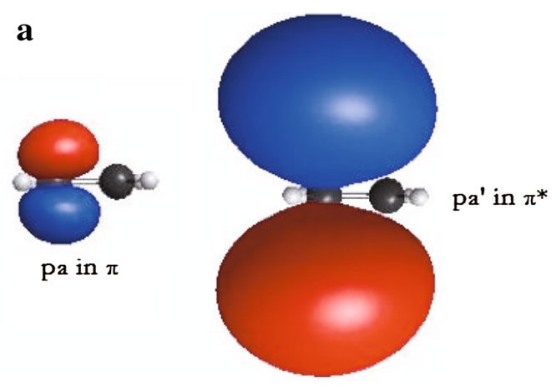

b
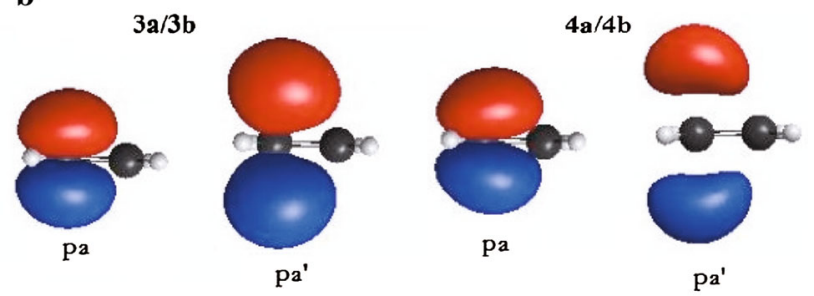

Fig. 1 The $\pi$ AOs of the $V$ state of ethylene, as calculated at the $\operatorname{CASSCF}(2,2)$ level (a upper figure), and at the dynamically correlated BOVB-5 level (b lower figure), showing the contraction of $\pi$ AOs under the effect of the dynamic $\sigma$ response to the fluctuation of $\pi$ electrons. All calculations in aug-VTZ basis set. The isodensity value is 0.045 a.u. in both (a) and (b) 
more instructive to consider this energy difference in a basis set like cc-pVTZ, where the valence-Rydberg mixing does not take place, for lack of diffuse basis functions. Thus, comparing entry 4 versus entry 6 in Table 2 yields the value $0.91 \mathrm{eV}$, which can be considered as an energetic measure of the importance of $\sigma$ response to the fluctuation of $\pi$ electrons, more precisely the greater importance of this $\sigma$ response in the $V$ state relative to the $N$ state.

Another outcome of the above VB calculations is the novel finding that structures $\mathbf{4 a}$ and $\mathbf{4 b}$, which are essentially asymmetric covalent structures, are essential ingredients of the $V$ state of ethylene. In terms of energy, their importance can be estimated as the difference between the 2-structure and 4-structure wave functions, and can be seen to be quite large at both the VBSCF and BOVB levels. At the BOVB level, the BOVB-3 versus BOVB-5 energy differences amount to $0.71,0.96$, and $0.92 \mathrm{eV}$ in $6-31 \mathrm{G}^{*}$, cc-pVTZ, and aug-VTZ basis sets, respectively. This significant stabilization can be interpreted as due to two effects: (1) a simple resonance energy, arising from the mixing of $4 \mathbf{a}, 4 \mathbf{b}$ with $\mathbf{3 a}, \mathbf{3 b}$, and (2) the tempering of the charge separation in the $\pi$ system in $\mathbf{3 a}$ and $\mathbf{3 b}$ when $\mathbf{4 a}$ and 4b are added. It is also remarkable that without structures 4a and $\mathbf{4 b}$, i.e., at the VBSCF-3 and BOVB-3 levels, the spatial extension of the wave function comes out too small, even in aug-VTZ basis set, with an $\left\langle x^{2}\right\rangle$ value of only 13.04 $\mathrm{a}_{0}^{2}$ in the $V$ state at the BOVB-3 level. Thus, it appears that the zwitterionic structures $\mathbf{3 a}$ and $\mathbf{3 b}$ have no physical reason to be very diffuse because the corresponding VB structures are overall neutral with positive charges in the vicinity of the negative ones. On the other hand, $\mathbf{4 a}$ and $\mathbf{4 b}$ must have diffuse orbitals, so as to clearly differentiate the two $\pi$ AOs involved in the covalent bond; otherwise, the negative combination $\mathbf{4 a - 4 b}$ would just vanish (see the above VB expansion). Thus, the reason for the known fact that the $V$ state is $50 \%$ more diffuse than the $N$ state does not lie in the zwitterionic structures, but in the asymmetrical covalent ones, $\mathbf{4 a}$ and $\mathbf{4 b}$.

In view of the essential role played by $\mathbf{4 a}$ and $\mathbf{4 b}$ in the electronic structure of the $V$ state, one may anticipate significant weights for these structures. However, the calculation of their weights can only be approximate owing to their large overlaps with $\mathbf{3 a}$ and $\mathbf{3 b}$ (Table 4). These large overlaps originate in the fact that all these structures involve diffuse AOs. For this reason, the usual CoulsonChirgwin definition of weights [47], which is quite sensitive to overlap [61], is better replaced by the two alternative definitions of the weights, Löwdin's scheme [48] and the "inverse-overlap" formula of Norbeck and Gallup [49]. These two definitions have been applied to calculate the weights of $\mathbf{3 a}, \mathbf{3 b}, \mathbf{4 a}$, and $\mathbf{4 b}$, which are displayed in Table 5 together with their coefficients in the BOVB-5 wave function of the $V$ state. The Löwdin and inverse- overlap weights are quantitatively different from each other, as always happens when the VB structures have strong mutual overlaps, and therefore, they can only provide orders of magnitude. Still, the calculated weights show that the major structures are $\mathbf{3 a}$ and $\mathbf{3 b}$, whereas $\mathbf{4 a}$ and $\mathbf{4 b}$ are less important but far from being negligible.

\subsection{VB calculations in the $(4,4)$ space}

Extending the space of VB structures so as to include the $\mathrm{C}-\mathrm{C} \sigma$ bond into the VB treatment leads to the 15 structures displayed in Scheme 3. Calculations in this full space (5-12b) are referred to as VBSCF-15 and BOVB-15. However, being redundant with 5-6b, structures 10a-12b are removed from the calculation of the $N$ state, while for obvious symmetry, reasons $\mathbf{5}$ is removed for the calculation of the $V$ state. Thus, the calculation of the $N$ and $V$ states involves 9 structures and 14 structures, respectively.

Since both the $\pi$ system and the $\sigma \mathrm{C}-\mathrm{C}$ bond are now involved in the VB treatment, one may expect the $\sigma$ response to fluctuation of the electrons to be present already at the VBSCF level, at least to some extent. This $\sigma$ response will be made apparent by comparing the weights of structures $8 \mathbf{a}$ versus $9 \mathbf{a}$, or $\mathbf{8 b}$ versus $9 \mathbf{b}$. If the two $\pi$ electrons are on the same AO, say the left one as in 7a-9a (Scheme 3), the $\sigma \mathrm{C}-\mathrm{C}$ bond will compensate this charge separation by polarizing itself in the opposite way, through the ionic structures $\mathbf{8 a}$ and $9 \mathbf{a}$. Therefore, one expects the weight of $\mathbf{8 a}$, in which both carbon atoms are globally neutral, to be significantly larger that that of $\mathbf{9 a}$, in which the carbons have formal charges of -2 and +2 , respectively. Inspection of Table 6 shows that this is indeed the case, as the weights of $8 \mathbf{a}$ and $9 \mathbf{a}$ are calculated to be 0.129

Table 4 The overlap matrix of $V$ state from BOVB-5 calculations with the aug-VTZ basis set in $(2,2)$ active space

\begin{tabular}{lllll}
\hline & 3a & 3b & 4a & 4b \\
\hline 3a & 1.000000 & & & \\
$\mathbf{3 b}$ & 0.316388 & 1.000000 & & \\
$\mathbf{4 a}$ & 0.845642 & 0.429452 & 1.000000 & \\
$\mathbf{4 b}$ & 0.429452 & 0.845641 & 0.474833 & 1.000000 \\
\hline
\end{tabular}

Table 5 The weights and coefficients of VB structures of the $V$ state from BOVB-5 calculations with the aug-VTZ basis set in $(2,2)$ active space

\begin{tabular}{lllr}
\hline & Löwdin weights & Inverse weights & Coefficients \\
\hline 3a & 0.4081 & 0.3047 & 1.1882 \\
3b & 0.4081 & 0.3047 & -1.1882 \\
4a & 0.0919 & 0.1953 & -0.9733 \\
4b & 0.0919 & 0.1953 & 0.9733 \\
\hline
\end{tabular}


Table 6 The weights and coefficients of VB structures for the $V$ state of ethylene, calculated with the aug-VTZ basis set in $(4,4)$ active space

\begin{tabular}{|c|c|c|c|c|c|c|}
\hline & \multicolumn{3}{|l|}{ VBSCF-15 } & \multicolumn{3}{|l|}{ BOVB-15 } \\
\hline & Löwdin weights & Inverse weights & Coefficients & Löwdin weights & Inverse weights & Coefficients \\
\hline $6 \mathbf{a}$ & 0.0003 & 0.0086 & 0.0437 & 0.0000 & 0.0130 & -0.0573 \\
\hline $6 b$ & 0.0003 & 0.0086 & -0.0437 & 0.0000 & 0.0130 & -0.0573 \\
\hline $7 a$ & 0.1786 & 0.1707 & 0.9472 & 0.1984 & 0.2391 & 0.8064 \\
\hline $8 \mathbf{a}$ & 0.1281 & 0.1028 & 0.4935 & 0.1149 & 0.0556 & 0.2672 \\
\hline $9 a$ & 0.0656 & 0.0013 & 0.0551 & 0.0915 & 0.0141 & 0.1378 \\
\hline $7 b$ & 0.1788 & 0.1710 & -0.9483 & 0.1984 & 0.2391 & -0.8064 \\
\hline $8 b$ & 0.1277 & 0.1018 & -0.4911 & 0.1149 & 0.0556 & -0.2672 \\
\hline $9 b$ & 0.0660 & 0.0014 & -0.0567 & 0.0915 & 0.0142 & -0.1378 \\
\hline $10 \mathrm{a}$ & 0.0624 & 0.1387 & -0.8696 & 0.0471 & 0.1376 & -0.6180 \\
\hline $11 \mathrm{a}$ & 0.0259 & 0.0009 & -0.0470 & 0.0248 & 0.0110 & -0.1172 \\
\hline $12 \mathbf{a}$ & 0.0388 & 0.0768 & -0.4400 & 0.0233 & 0.0297 & -0.1919 \\
\hline $10 \mathrm{~b}$ & 0.0624 & 0.1389 & 0.8705 & 0.0471 & 0.1376 & 0.6180 \\
\hline $11 b$ & 0.0260 & 0.0009 & 0.0484 & 0.0248 & 0.0110 & 0.1172 \\
\hline $12 b$ & 0.0390 & 0.0776 & 0.4423 & 0.0233 & 0.0297 & 0.1919 \\
\hline
\end{tabular}

Table 7 The vertical excitation energy in $(4,4)$ active space, as calculated by VB methods with the aug-VTZ basis set

\begin{tabular}{|c|c|c|c|c|c|}
\hline & \multicolumn{2}{|l|}{$N$ state } & \multicolumn{2}{|l|}{$V$ state } & \multirow{2}{*}{$\begin{array}{l}T_{\mathrm{e}} \\
(\mathrm{eV})\end{array}$} \\
\hline & E (a.u.) & $\begin{array}{l}\left\langle x^{2}\right\rangle \\
\left(\mathrm{a}_{0}^{2}\right)\end{array}$ & E (a.u.) & $\begin{array}{l}\left\langle x^{2}\right\rangle \\
\left(a_{0}^{2}\right)\end{array}$ & \\
\hline VBSCF-9 $9^{a}$ & -78.110778 & 11.68 & -77.769936 & 13.44 & 9.27 \\
\hline VBSCF- $15^{\mathrm{b}}$ & -78.110778 & 11.68 & -77.811182 & 22.95 & 8.15 \\
\hline $\operatorname{CASSCF}(4,4)$ & -78.112095 & 11.60 & -77.800412 & 22.21 & 8.48 \\
\hline BOVB- $9^{\mathrm{a}}$ & -78.121389 & 11.76 & -77.796188 & 13.07 & 8.85 \\
\hline BOVB- $15^{\mathrm{b}}$ & -78.121389 & 11.76 & -77.828470 & 19.16 & 7.97 \\
\hline
\end{tabular}

${ }^{\text {a }}$ Involves structures $\mathbf{5}-\mathbf{9 b}$ for the $N$ state, and $\mathbf{6 a}-\mathbf{9 b}$ for the $V$ state

${ }^{\mathrm{b}}$ Involves structures 5-9b for the $N$ state, and $\mathbf{6 a - 1 2 b}$ for the $V$ state

and 0.066 at the VBSCF-15 level, according to Löwdin's definition of the weights. Moreover, the difference in weights is even more significant with the inverse-overlap weight definition. Of course, similar differences in weights are found between structures $\mathbf{8 b}$ and $\mathbf{9 b}$.

It should be noted that the $\sigma$ response to $\pi$ fluctuation, as it appears from the weights displayed in Table 6, is incomplete at the VBSCF-15 level since it is restricted to the $\sigma \mathrm{C}-\mathrm{C}$ bond and does not involve the $\mathrm{C}-\mathrm{H}$ bonds. Still, one expects some improvement of the description of the $V$ state with respect to the VBSCF calculations in the $(2,2)$ space. Table 7 reports the absolute energies of the $N$ and $V$ states as calculated in the $(4,4)$ space, together with their spatial extensions and the $N \rightarrow V$ transition energies $T_{e}$. While the CASSCF results are still poor at this level, it can be seen that the VBSCF-15 results are indeed improved relative to the VBSCF-5 results in the $(2,2)$ space
(Table 3), as the $\left\langle x^{2}\right\rangle$ value goes down from 24.92 to $22.95 \mathrm{a}_{0}^{2}$, while the transition energy also goes down, from 8.26 to $8.15 \mathrm{eV}$. However, in spite of these improvements, the results are not fully satisfying at this level, and once again, one must use the BOVB level in the same space to retrieve the missing dynamic $\sigma \pi$ correlation. To facilitate convergence, only partial BOVB is performed, in which the VB structures are partitioned into groups: $(\mathbf{5}-\mathbf{6 b}),(\mathbf{7} \mathbf{a}-$ 9a), (7b-9b), (10a-12a), and (10b-12b). A common set of orbitals is used within each group, but orbitals of one group are allowed to be different from those of other groups. The results are now excellent, with a spatial extension of $19.16 \mathrm{a}_{0}^{2}$ for the $V$ state, and a transition energy of $7.97 \mathrm{eV}$, only $0.09 \mathrm{eV}$ higher than the reference value of $7.88 \mathrm{eV}$. Interestingly, these latter results are virtually unchanged relative to the BOVB-5 results in the $(2,2)$ space, showing that all the necessary electron correlation is already taken into account in the smaller VB space.

Since VB structures displaying asymmetrical $\pi$ covalent bonds proved essential for the description of the $V$ state in the $(2,2)$ space, one may wonder if structures of the same type $(\mathbf{1 0 a}-\mathbf{1 2 b})$ have a comparable importance in the $(4,4)$ space. Clearly, the answer is yes: In terms of weights, structures 10a-12b sum up to 0.190 with the Löwdin definition, and to 0.357 with the inverse-overlap one (Table 5, BOVB-15). This can be compared with the cumulated weights 0.184 and 0.391 , respectively, with the same definitions, in the $(2,2)$ space (Table 5). In terms of stabilization energies, the stabilization brought by structures 10a-12b can be estimated by comparing the calculations of the $V$ state in the 15-structure space (entries 2 and 5 in Table 7), to those using a restricted 9-structure space, 
Table 8 Energies of the $N$ and $V$ states and weights of structures $\mathbf{4 a}$ and $\mathbf{4 b}$, as calculated at the VMC and DMC levels using VBSCF or BOVB wave functions as trial functions, with the ps-aug-VTZ basis set

\begin{tabular}{|c|c|c|c|c|c|}
\hline \multirow[t]{2}{*}{ QMC method } & \multicolumn{2}{|l|}{$N$ state } & \multicolumn{2}{|l|}{$V$ state } & \multirow[t]{2}{*}{$T_{\mathrm{e}}(\mathrm{eV})$} \\
\hline & Optimized parameter ${ }^{\mathrm{a}}$ & E (a.u.) & E (a.u.) & Weigths of $4 a, 4 b^{b}$ & \\
\hline $\mathrm{VB}-\mathrm{VMC}-5^{\mathrm{c}, \mathrm{d}}$ & $\mathrm{j}+\mathrm{c}$ & $-13.7132(5)$ & $-13.3977(5)$ & $0.138(1)$ & $8.59(2)$ \\
\hline $\mathrm{BO}-\mathrm{VMC}-5^{\mathrm{c}, \mathrm{e}}$ & $\mathrm{j}+\mathrm{c}$ & $-13.7053(5)$ & $-13.4098(5)$ & $0.092(1)$ & $8.04(2)$ \\
\hline BO-VMC-5 $5^{\mathrm{c}, \mathrm{e}}$ & $\mathrm{j}+\mathrm{c}+\mathrm{i}$ & $-13.7221(5)$ & $-13.4294(5)$ & $0.074(1)$ & $7.96(2)$ \\
\hline BO-VMC- $3^{\mathrm{c}, \mathrm{e}, \mathrm{f}}$ & $\mathrm{j}+\mathrm{c}$ & $-13.7053(5)$ & $-13.3751(5)$ & - & $8.98(2)$ \\
\hline BO-DMC-5 $5^{\mathrm{c}, \mathrm{e}}$ & $\mathrm{j}+\mathrm{c}$ & $-13.7382(2)$ & $-13.4455(2)$ & $0.087(1)$ & $7.96(1)$ \\
\hline BO-DMC-5 $5^{\mathrm{c}, \mathrm{e}}$ & $\mathrm{j}+\mathrm{c}+\mathrm{i}$ & $-13.7439(2)$ & $-13.4525(2)$ & $0.074(<1) \%$ & $7.93(1)$ \\
\hline
\end{tabular}

Statistical errors are shown in parenthesis

${ }^{a}$ Parameters optimized at the VMC level: j for Jastrow parameters, c for CSFs coefficients, i for inactive $(\sigma)$ orbitals

${ }^{b}$ Weights of each of the structures, Löwdin definition, Eq. 3

${ }^{\mathrm{c}}$ Involves structures 2, 3a, and $\mathbf{3 b}$ for the $N$ state, and $\mathbf{3 a}, \mathbf{3 b}, \mathbf{4 a}, \mathbf{4 b}$ for the $V$ state

${ }^{d}$ A VBSCF wave function is used as a trial function

e A BOVB wave function is used as a trial function

${ }^{\mathrm{f}}$ Involves structures $\mathbf{2}, \mathbf{3 a}$, and $\mathbf{3 b}$ for the $N$ state, and only $\mathbf{3 a}, \mathbf{3 b}$ for the $V$ state

involving only structures 5-9b (entries 1 and 4). The stabilization amounts to 1.12 and $0.88 \mathrm{eV}$, respectively, at the VBSCF and BOVB level. Moreover, as was also found in the $(2,2)$ space, the spatial extension of the $V$ state comes out too small (ca. $13 \mathrm{a}_{0}^{2}$ ) if structures $\mathbf{1 0 a}-\mathbf{1 2 b}$ are omitted. Thus, from all point of views, it appears that the newly proposed VB structures displaying asymmetrical $\pi$ covalent bonds are essential ingredients of the $V$ state of ethylene, albeit they were never considered before, to our knowledge.

\subsection{VB-QMC calculations}

Since QMC methods are known to be extremely accurate in principle, provided one uses sufficiently good trial functions, it is interesting to check whether the extra correlation provided by these methods would change or confirm our above results. To this aim, we have used the QMC methods by inputting the above VBSCF and BOVB wave functions as trial wave functions, in so-called VB-QMC methods [44]. Two levels of VB-QMC calculations have been used. At the VMC level, previously optimized VBSCF or BOVB wave functions are supplemented with a Jastrow factor including explicit interparticle correlation (see theoretical section), and wave function parameters are reoptimized. Then, the more accurate DMC level consists of projecting the VMC wave function onto the ground state using the fixed-node DMC algorithm.

Table 8 displays the results obtained at these two levels, where VB-VMC (resp. BO-DMC) corresponds to a wave function that has a VBSCF (resp. BOVB) type of determinantal part. It can be seen that when a simple VBSCF wave function is used as a trial function (VB-VMC, entry $1)$, the $N \rightarrow V$ transition energy is disappointingly large, $8.59 \mathrm{eV}$, showing that the Jastrow factor is not able to retrieve the $\sigma \pi$ dynamic polarization that accompanies the fluctuation of the $\pi$ electrons. On the other hand, using now the BOVB-5 wave function as a trial function, one gets a much better $T_{\mathrm{e}}$ value of $8.04 \mathrm{eV}$ when only the Jastrow parameters and coefficients of the CSFs are optimized (entry 2), and an even better value, $7.96 \mathrm{eV}$, if a further optimization of the inactive orbitals is performed (entry 3 ). By contrast, removing structures $\mathbf{4 a}$ and $\mathbf{4 b}$ from the set of VB structures in the trial function, as in the BO-VMC-3 calculation, leads to a much too high $N \rightarrow V$ transition energy, $8.98 \mathrm{eV}$. This result further confirms that structures 4a and $4 \mathbf{b}$ are essential components of the $V$ state of ethylene, even when a high level of electron correlation is brought by the Jastrow factor.

Fixed-node DMC calculations further improve the BOVMC-5 results. Using a BOVB-5 trial wave function where only Jastrow and CSFs parameters were reoptimized at the VMC level leads to a DMC transition energy of $7.96 \mathrm{eV}$, quite close to the experimental value, in excellent agreement with experiment $(7.88 \mathrm{eV})$, showing that the nodal structure of the standard BOVB level is physically correct. This has to be compared with the best DMC value of $8.27 \mathrm{eV}(190.8 \mathrm{kcal} / \mathrm{mol})$ obtained by Anderson et al. [32], using a GVB-type trial function. Finally, using the BOVB-5 trial wave function where the $\sigma$ orbitals were also reoptimized at the VMC level further improves the transition energy to $7.93 \mathrm{eV}$, identical within the error bars to the DMC value obtained by Schautz and Filippi [31]. 


\section{Conclusions}

The $V$ state of ethylene poses a long-standing challenge for theoretical methods, despite the small size of the molecule. The difficulties encountered with standard MO-CI methods are due to (1) the strong charge fluctuation within the $\pi$ system, which requires a dynamic response of the $\sigma$ frame already at the orbital optimization step, and (2) a low-lying Rydberg state which may spuriously contaminate the $V$ state. Thus, any meaningful wave function for the $V$ state must satisfy the dual condition of providing an accurate energy relative to the ground state, and a reasonable spatial extension, neither too large nor too small, i.e., being about $50 \%$ larger than that of the ground state but not more.

The strategies of overcoming these difficulties in the MO framework are all based on simple VB reasoning. These methods are generally successful, but lead to long configuration expansions that are treated by multi-reference variational or perturbative methods. This obviously suggests the use of direct VB treatments, which are performed with two different methods, VBSCF that is limited to static electron correlation (like GVB and SC methods), and BOVB that further involves dynamic correlation while keeping the number of VB structures unchanged. At the VBSCF level, the results share the same shortcomings as CASSCF calculations and provide too large $N \rightarrow V$ transition energies and $V$ state wave functions that are too diffuse to qualify as valence state. On the other hand, the BOVB level provides excellent results in terms of both transition energies and spatial extension of the wave functions. The number of configurations amounts to only 14 structures in the $(4,4)$ VB space, and 4 VB structures in the $(2,2)$ space. Both VB structure sets lead to practically identical results, showing that $4 \mathrm{VB}$ structures with the BOVB method are already capable of taking care of the $\sigma$ response to the fluctuations of the $\pi$ electrons, by means of the breathing orbital effect. Moreover, the contraction of the $\pi$ AOs under the influence of dynamic electron correlation can be clearly visualized by turning this latter type of correlation on and off, by switching from BOVB to VBSCF.

The BOVB wave functions of the $(2,2)$ space have also been used as guess functions for diffusion Monte Carlo calculations, yielding results in close agreement with the calculations of Schautz and Filippi [31], even without needing to re-optimize the guess orbitals at the VMC step. This further confirms the adequacy of BOVB wave functions as guesses for DMC calculations, as noted already in previous studies [44, 62].

Another outcome of the present study is the evidence for the importance of VB structures displaying an asymmetric $\pi$ covalent bond as an essential component of the $V$ state of ethylene. Such VB structures, which have never been considered before in qualitative descriptions of the $V$ state, arise naturally from an expansion of the $\pi \pi^{*}$ MO configuration into VB structures. These asymmetric $\pi$-bonds contribute $18-26 \%$ of the total VB wave function. Moreover, they have quite a significant stabilizing effect, of the order of ca. $0.90 \mathrm{eV}$, which can be understood as their role in tempering the fluctuations of the $\pi$ electrons, together with a classical resonance effect due to their mixing with the zwitterionic structures. Lastly, the two asymmetric covalent VB structures are the ones responsible for the spatial extension of the $V$ state being $50 \%$ larger than that of the $N$ state. It must therefore be concluded that the $V$ state of ethylene is not $100 \%$ ionic as usually assumed, but involves a minor though non-negligible covalent component. Incidentally, the disregard of these structures in current qualitative reasoning is due to the usage of a minimal basis set in the decomposition of the $\pi \pi^{*}$ configuration, in which the asymmetrical covalent structures vanish. Nevertheless, the importance of these structures does not imply that carbon has suddenly five valence orbitals in the $V$ state. The effect of $\mathbf{4 a}$ and $\mathbf{4 b}$ is essentially perturbational, and it dresses the zwitterionic structures with left-right correlation, which tempers the antiresonant interaction (of the $\mathbf{3} \mathbf{a}-\mathbf{3 b}$ combination in the minimal VB set) and the high electron-electron repulsion of the doubly occupied $2 p$ orbitals. It is tempting to assign the diffuse orbitals in $\mathbf{4 a}$ and $\mathbf{4 b}$ as carbon $3 p$ AOs, however, we were not able to verify this character since the orbitals lack the requisite radical node to qualify as $3 p$.

All in all, the above study shows that performing direct VB calculations in a reasonable basis set may lead to an accurate description of the $V$ state by means of extremely compact wave functions provided the VB treatment involves a built-in dynamic electron correlation. The reason for the success is that VB can achieve this in a direct way, whereas MO-CI methods must do this in a roundabout and sometimes complicated way, owing to orthogonality constraints. By contrast, the choice of VB structures that need be involved in the computations does not require complicated reasoning, but naturally arises from chemical considerations. The additional and sometimes unexpected insight that arises from such calculations is an intrinsic feature of modern ab initio VB methods.

Acknowledgments W. W. is supported by the Natural Science Foundation of China (Nos. 21120102035, 21273176, 21290193). SS thanks the Israel Science Foundation (ISF grant 1183/13). B. B. thanks the IDRIS computational center for an allocation of computer time.

\section{References}

1. Cooper DL, Gerratt J, Raimondi M (1991) Chem Rev 91:929

2. Gerratt J, Cooper DL, Karadakov PB, Raimondi M (1998) Spincoupled theory. Wiley, New York 
3. Goddard WA, Dunning TH Jr, Hunt WJ, Hay PJ (1973) Acc Chem Res 6:368

4. Bobrowicz FW, Goddard WA (1977) In: Schaefer III HF (ed) Methods of electronic structure theory, vol 4. Springer, Heidelberg, p 79

5. Hiberty PC, Shaik S (2007) J Comput Chem 28:137

6. Shaik S, Hiberty PC (2008) A chemist's guide to valence bond theory. Wiley, Hoboken

7. Wu W, Su P, Shaik S, Hiberty PC (2011) Chem Rev 111:7557-7593

8. Shaik S, Hiberty PC (2004) Rev Comp Chem 20:1-100

9. Hiberty PC, Humbel S, van Lenthe JH, Byrman C (1994) J Chem Phys 101:5969

10. Hiberty PC, Flament J-P, Noizet E (1992) Chem Phys Lett 189:259

11. Hiberty PC, Shaik S (2002) Theoret Chem Acc 108:255

12. Angeli C (2009) J Comput Chem 30:1319

13. Merer AJ, Mulliken RS (1969) Chem Rev 69:639

14. Wilkinson G, Mulliken RS (1955) J Chem Phys 23:1895

15. Dunning TH Jr, Hunt WJ, Goddard WA (1969) Chem Phys Lett 4:147

16. Brooks BR, Schaefer HF III (1975) Chem Phys 9:75

17. Schaefer HF III (1978) J Chem Phys 68:4839

18. Buenker RJ, Shih S-K, Peyerimhoff SD (1979) Chem Phys 36:97

19. Buenker RJ, Peyerimhoff SD, Shih S-K (1980) Chem Phys Lett 69:7-13

20. Bender CF, Dunning TH Jr, Schaefer HF III, Goddard WA, Hunt WJ (1972) Chem Phys Lett 15:171

21. Buenker RJ, Peyerimhoff SD (1975) Chem Phys 9:75

22. McMurchie LE, Davidson ER (1977) J Chem Phys 66:2959

23. Duben AJ, Goodman L, Pamuk HO, Sinanoglu O (1973) Theoret Chim Acta (Berl) 30:177

24. Sinanoglu O (1969) Adv Chem Phys 14:237

25. Davidson ER (1996) J Phys Chem 100:6161

26. Davidson ER, Jarzecki A (1998) Chem Phys Lett 285:155

27. Lasorne B, Jornet-Somoza J, Meyer H-D, Lauvergnat D, Robb MA, Gatti F (2013) Spect Acta A. http://dx.doi.org/10.1016/j.saa. 2013.04.078

28. Finley J, Malmqvist P-Å, Roos BO, Serrano-Andrès L (1998) Chem Phys Lett 288:299

29. Krebs S, Buenker RJ (1997) J Chem Phys 106:7208

30. Müller T, Dallos M, Lischka H (1999) J Chem Phys 110:7176

31. Schautz F, Filippi C (2004) J Chem Phys 120:10931

32. Anderson AG, Goddard WA (2010) J Chem Phys 132:164110

33. Zaitsevskii A, Malrieu J-P (1995) Int J Quantum Chem 55:117-125

34. Garcia-Cuesta I, Sanchez de Meras AMJ, Koch H (2003) J Chem Phys 118:8216
35. Watts JD, Gwaltney SR, Bartlett RJ (1996) J Chem Phys 105:6979

36. Wu W, Song L, Cao Z, Zhang Q, Shaik S (2002) J Phys Chem A 106:2721

37. Song L, Wu W, Zhang Q, Shaik S (2004) J Comput Chem 25:472

38. Chen Z, Song J, Shaik S, Hiberty PC, Wu W (2009) J Phys Chem A 113:11560

39. Shaik S, Danovich D, Silvi B, Lauvergnat D, Hiberty PC (2005) Chem Eur J 11:6358

40. Hiberty PC, Humbel S, Archirel P (1994) J Phys Chem 98:11697

41. Song L, Wu W, Hiberty PC, Danovich D, Shaik S (2003) Chem Eur J 9:4540

42. Hiberty PC, Megret C, Song L, Wu W, Shaik S (2006) J Am Chem Soc 128:2836

43. Su P, Song L, Wu W, Hiberty PC, Shaik S (2007) J Comput Chem 28:185

44. Braida B, Toulouse J, Caffarel M, Umrigar CJ (2011) J Chem Phys 134:084108

45. Braida B, Hiberty PC (2013) Nat Chem 5:417

46. Toulouse J, Umrigar CJ (2008) J Chem Phys 128:174101

47. Chirgwin HB, Coulson CA (1950) Proc R Soc Lond Ser A 201:196

48. Löwdin PO (1947) Ark Mat Astr Fysik 35A:9

49. Gallup GA, Norbeck JM (1973) Chem Phys Lett 21:495

50. van Lenthe JH, Balint-Kurti GG (1983) J Chem Phys 78:5699

51. Dunning TH Jr (1989) J Chem Phys 90:1007

52. Kendall RA, Dunning TH Jr, Harrison RJ (1992) J Chem Phys 96:6796

53. Petruzielo FR, Toulouse J, Umrigar CJ (2012) J Chem Phys 136:124116

54. Burkatzki M, Filippi C, Dolg M (2007) J Chem Phys 126, 234105. Basis sets and corresponding pseudopotentials are available online: http://burkatzki.com/pseudos/index.2.html

55. Song L, Wu W, Mo Y, Zhang Q (2003) XMVB: an ab initio Nonorthogonal Valence Bond Program, Xiamen University, Xiamen 361005, China

56. Song L, Mo Y, Zhang Q, Wu W (2005) J Comput Chem 26:514

57. Song L, Song J, Mo Y, Wu W (2009) J Comput Chem 30:399

58. Umrigar CJ, Filippi C, Toulouse J. CHAMP, a Quantum Monte Carlo ab initio program, see: http://pages.physics.cornell.edu/ $\sim$ cyrus/champ.html

59. Lindh R, Roos BO (1989) Int J Quantum Chem 35:813

60. Serrano-Andrès L, Merchan M, Nebot-Gil I, Roos BO (1993) J Chem Phys 98:3151

61. Thorsteinsson T, Cooper DL (1998) J Math Chem 23:105-126

62. Domin D, Braida B, Lester WA Jr (2008) J Phys Chem A 112:8964 